\title{
$A b$ Initio Density Functional Theory Investigation of the Interaction between Carbon Nanotubes and Water Molecules during Water Desalination Process
}

\author{
Loay A. Elalfy, ${ }^{1}$ Walid M. I. Hassan, ${ }^{2}$ and Wael N. Akl ${ }^{1,3}$ \\ ${ }^{1}$ Center for Nanotechnology, Nile University, B2, Smart Village, Km 28 Cairo-Alex Desert Road, Cairo 12677, Egypt \\ ${ }^{2}$ Department of Chemistry, Faculty of Science, Cairo University, Cairo 12613, Egypt \\ ${ }^{3}$ Design and Production Engineering Department, Faculty of Engineering, Ain Shams University, Cairo 11566, Egypt \\ Correspondence should be addressed to Walid M. I. Hassan; walid_m76@yahoo.com
}

Received 31 May 2013; Accepted 3 September 2013

Academic Editor: Yang Xu

Copyright (C) 2013 Loay A. Elalfy et al. This is an open access article distributed under the Creative Commons Attribution License, which permits unrestricted use, distribution, and reproduction in any medium, provided the original work is properly cited.

\begin{abstract}
Density functional theory calculations using B3LYP/3-21G level of theory have been implemented on 6 carbon nanotubes (CNTs) structures (3 zigzag and 3 armchair CNTs) to study the energetics of the reverse osmosis during water desalination process. Calculations of the band gap, interaction energy, highest occupied molecular orbital, lowest unoccupied molecular orbital, electronegativity, hardness, and pressure of the system are discussed. The calculations showed that the water molecule that exists inside the CNT is about 2-3 $\AA$ away from its wall. The calculations have proven that the zigzag CNTs are more efficient for reverse osmosis water desalination process than armchair CNTs as the reverse osmosis process requires pressure of approximately $200 \mathrm{MPa}$ for armchair CNTs, which is consistent with the values used in molecular dynamics simulations, while that needed when using zigzag CNTs was in the order of $60 \mathrm{MPa}$.
\end{abstract}

\section{Introduction}

Fresh water scarcity worldwide is growing to be one of the most critical issues facing the development of mankind. In the last two decades, more research has been done to develop new efficient techniques for water desalination [1-3], since the current approaches are energy consuming ones, which is another challenge facing the human race. The use of new materials became a trend in dealing with such problem. The reason for using carbon nanotubes (CNTs) in water desalination processes and other applications is due to their wide range of electronic, magnetic, chemical, biological, and mechanical properties that depend on their chirality [4-7].

The CNT $[8,9]$ is formed from wrapped sheet of graphene whose unit cell is composed of 2 carbon atoms with 2 translational unit vectors $a_{1}$ and $a_{2}$ forming an included angle of 30 degrees, where the edge of any CNT is a wrapped vector formed of a linear combination of these vectors. Graphene has $\mathrm{sp}^{2}$ hybridized carbon atom that makes strong $\sigma$ bonds with the other 3 identical carbon atoms at an angle of 120 leaving a weekly $\pi$ bonded $p_{z}$ electrons. This leads to the formation of an electron cloud on the wall of the tube which is the active component of the CNT. The adsorption of different molecules such as oxygen, hydrogen, and methane on CNTs is being studied. The adsorption of oxygen molecules and atoms are studied using density functional theory (DFT) [10], and it was found that oxygen tends to be physisorbed and chemisorbed on the inner and the outer walls of the CNTs, respectively. More studies were made on hydrogen - for hydrogen storage applications-using DFT and grand canonical Mote Carlo (GCMC) that showed the dependence of the adsorption process on the temperature and pressure [11-13]. A collective study was made on the adsorption of different gas molecules including water on CNTs from the outer side of the tube [14]. Many experimental trials have been made to employ such properties to the aim of water desalination by adding multiwall carbon nanotubes (MWCNTs) to microporous desalination membranes [15]. Pioneer molecular dynamics simulations for water conduction through CNT showed that 
spontaneous and continuous filling of CNT with a onedimensionally ordered chain of water molecules with a pulselike transmission of water through CNT [16]. Furthermore, molecular dynamics techniques have been implemented to calculate the flow rate of water through a reverse osmosis (RO) membrane formed of SWCNT array [17]. The most comprehensive study proved that such membrane would permit water to flow 600 times higher than the current commercial membranes with $100 \%$ salt rejection for the $(5,5)$ SWCNT and more than 1800 times with 95\% salt rejection for the $(7,7)$ SWCNT [18]. However, all of the aforementioned studies lack the description of the interaction between water molecules and CNT that is directly related to the flow rate of water.

In this study, detailed calculations of the energetics and dispersion interaction energy of a water molecule inside $(5,5),(6,6)$, and $(7,7)$ armchair CNTs and $(8,0),(10,0)$, and $(12,0)$ zigzag CNTs of radii ranging from $6.2 \AA$ to $9.5 \AA$ have been carried out. This aims to make a comprehensive understanding of the relationship between radius and flow rate of water inside the CNTs showing the energetically optimal region for water to exist inside the CNT. Eventually, such simulations would provide a better ground to assess the adequacy of the different CNT types to reverse osmosis water desalination process.

\section{Computational Method}

The initial molecular geometry for all CNTs was obtained by VMD [19] which is a molecular visualization program for displaying, animating, and analyzing large biomolecular systems using 3D graphics and built-in scripting. The water molecule geometry inside CNTs was fully optimized at the 3-21 G basis set using the Becke, three-parameter, Lee-YangParr hybrid (B3LYP) method [20-23]. The B3LYP method provides better energetics compared to that of Hartree-Fock and can reproduce better geometrical parameters comparable to the experimental values [24]. Even though using higher basis set is expected to enhance the accuracy of the energy calculation, such accuracy has been reported not to generally affect the simulation trends, yet consuming more computational power [10]. All the geometry optimization and energetics were done using the Gaussian 09 software package [25]. The optimized structures were visualized using GaussView version 5.0.9 package [26]. One-dimensional periodic boundary condition (PBC) along the CNT axis direction was employed. $\mathrm{PBC}$ calculations are usually utilized for periodic systems that have many repeating units such as polymers and crystalline minerals. $\mathrm{PBC}$ was used for the calculation of interaction of water molecules with CNT. One water molecule per unit cell was simulated in the tube axis direction. In the studied cases, the cell length was $2.456 \AA$ and $4.254 \AA$ for arm chair and zigzag structures, respectively.

The strength of adsorption/desorption depends on the interaction between the water molecule and the carbon atoms of the CNT. The $\mathrm{p}_{\mathrm{z}}$ electronic density of CNT is relatively high [27]. The polar water molecule is expected to disperse inside the tube to reach the position of lowest interaction with the tube, which is measured by optimizing the position of the water molecule inside the CNT. The geometry optimization process will be conducted starting from $1.5 \AA$ away from the inner wall of the CNT to evaluate the area where the water molecules flow freely. The interaction energy $\left(E_{\text {int }}\right)$ will be calculated as the difference between the energy of the $\mathrm{CNT}$ with the water molecule at the optimized geometry $\left(E_{\text {water+CNT }}\right)$ and the sum of the optimized energy of each molecule separately $\left(E_{\mathrm{CNT}}\right)$ and $\left(E_{\text {water }}\right)$ :

$$
E_{\text {int }}=E_{\text {water }+\mathrm{CNT}}-\left(E_{\mathrm{CNT}}+E_{\text {water }}\right) .
$$

The interaction energy will be used to calculate the pressure $(P)$ required to be applied on water in RO process for water to pass through the CNT. The pressure will be defined by statistical approach:

$$
P=\left(\frac{\partial F}{\partial V}\right)_{T, N},
$$

where $F$ is the Helmholtz free energy, $V$ is the volume containing the particles which will be given by the volume of 1 unit cell, $T$ is the temperature, and $N$ is the number of the particles.

The Helmholtz free energy $F$ can be defined as

$$
F=E-T S \text {, }
$$

where $S$ is the entropy of the system.

The differential form is

$$
d F=d E-T d S-S d T
$$

Since the adsorption/desorption process is reversible and isothermal (i.e., $d S, d T=0$ ), then

$$
P=\left(\frac{\partial E}{\partial V}\right)_{T, N} \cong \frac{E_{b}}{V} .
$$

Calculation of the electronegativity $(\chi)$ and hardness $(\eta)$ of the water and CNTs will be carried out to measure the tendency of the water molecules to interact with different types of CNT. Electronegativity is the tendency of molecule to acquire one more electron and involve in a new interaction [28]. The hardness of water and CNT gives an idea on how strong the interaction takes place between the two molecules, since hardness is defined in several ways such as "hard likes hard and soft likes soft" in the hard-soft-acid-base (HSAB) principle [29], and that the atoms arrange themselves in order to reach the maximum hardness according to the maximum hardness principle (MHP) [30]. The electronegativity is calculated as the negative half of the sum of the energies of the highest occupied molecular orbital (HOMO) and the lowest unoccupied molecular orbital (LUMO) [10]:

$$
\chi=\frac{\mathrm{HOMO}+\mathrm{LUMO}}{2},
$$

whereas the hardness is calculated as half the difference between LUMO and HOMO [16]:

$$
\eta=\frac{\mathrm{LUMO}-\mathrm{HOMO}}{2} .
$$



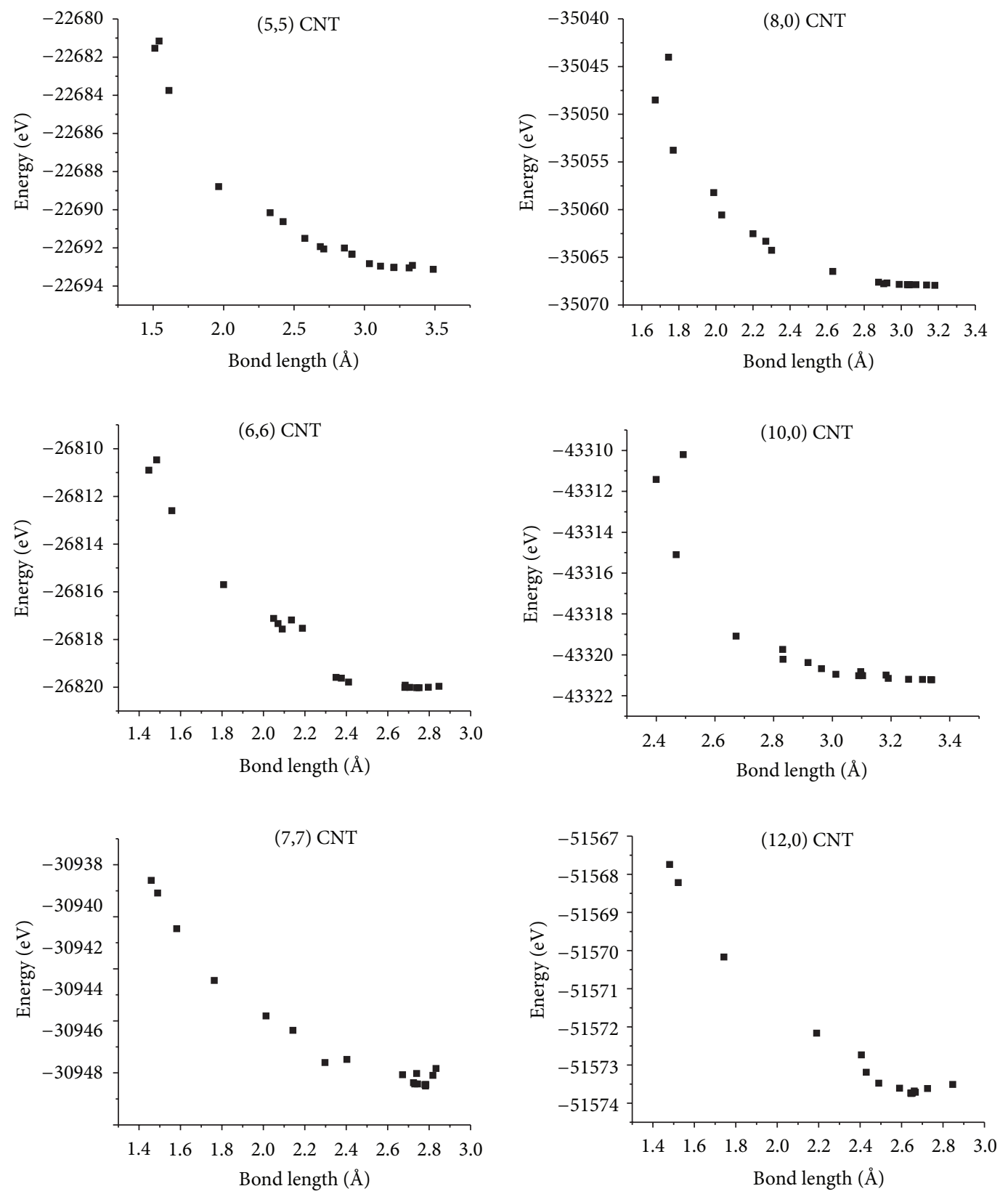

FIGURE 1: The energy versus the distance between water molecule and the CNT wall during the geometry optimization.

\section{Results and Discussion}

The simulation process started by optimizing the geometry and location of water molecule inside the CNTs. The energy versus water-wall distance during the optimization process is shown in Figure 1. In all configurations, the energy of the water molecule near the CNT wall was high and decreased exponentially to reach stable region few angstroms away from the wall depending on the CNT type or size. The optimization process showed that, at the neighborhood of the wall, there is a skin layer where water molecules suffer a strong repulsion, which is characterized by the point after which the energy of the system reaches saturation, and its thickness will be denoted by $(\lambda)$ and defined as the distance from the CNT wall with the energy $10 \%$ more than the optimized geometry energy.

This means the water molecule at a distance larger than $\lambda$ from the wall will be energetically more stable and can move with minimum repulsion with $\mathrm{CNT}$ inside this region. The area of this region will be called effective area $A_{\text {eff }}$ of the CNT and can be calculated by

$$
A_{\mathrm{eff}}=\pi\left(\left(\frac{d}{2}\right)-\lambda\right)^{2}
$$

where $d$ is the diameter of CNT. 


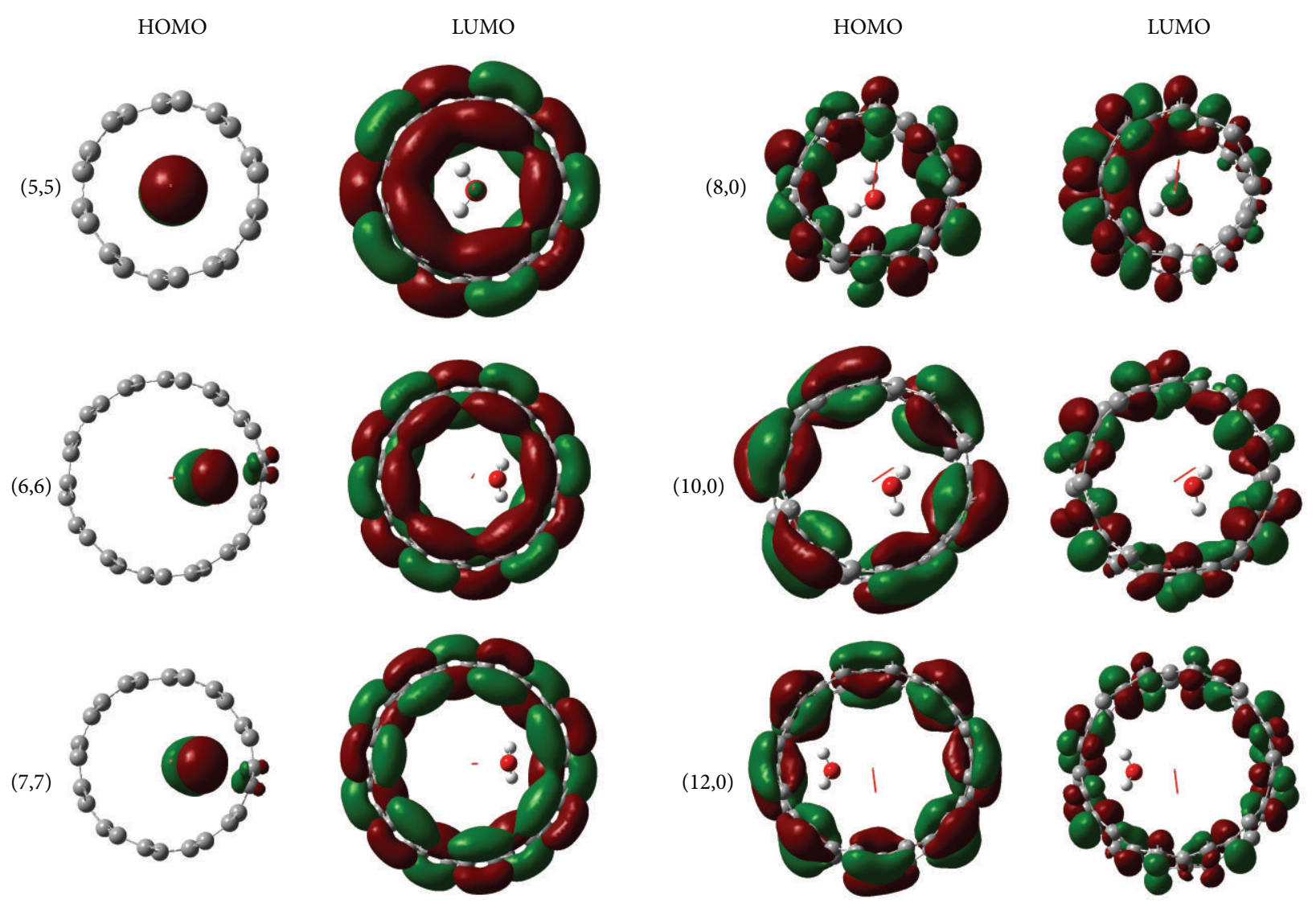

FIGURE 2: HOMO and LUMO of the CNTs and water at the optimized geometry.

The values of $A_{\text {eff }}$ are shown in Table 1 . As a general trend, $A_{\text {eff }}$ increases, but not linearly, with the increase of the CNT diameter.

The electronic configuration densities of the HOMO and the LUMO of CNT and water at optimized geometry are shown in Figure 2. The molecular orbital shows that in case of armchair, the HOMO is mainly due to the water molecule and that the LUMO is mainly due to CNT. The HOMO in $(5,5)$ CNT does not show any interaction between CNT and water orbital, whereas in case of $(6,6)$ and $(7,7) \mathrm{CNTs}$, a more clear interaction is evident. All the zigzag HOMO and LUMO CNTs show minimum interact between CNT and water molecule except for the LUMO of the $(8,0)$ CNT where strong interaction occurs which can be related to the smallest diameter of $(8,0)$ among all the studied CNTs.

At the optimized geometry, the energetics at the $\Gamma$ point is summarized in Table 2 for CNTs. Different shapes of CNTs have shown to possess almost the same electronegativity. The band gap increases with the CNT diameter in the armchair $\mathrm{CNT}$, while it decreases as the CNT diameter increases in zigzag CNT.

The optimized water molecule has a band gap of $9.357 \mathrm{eV}$ and a hardness $\eta$ of $4.679 \mathrm{eV}$. Armchair CNTs are much harder than zigzag CNTs. The hardness of armchair CNTs increases from $2.58 \mathrm{eV}$ to $2.75 \mathrm{eV}$ with the increase of the CNT diameter. The zigzag CNTs hardness is $0.8 \mathrm{eV}$ and $0.7 \mathrm{eV}$ for the $(8,0)$ and $(10,0)$, respectively, and moreover the hardness
TABle 1: The diameter, $\lambda$, and $A_{\text {eff }}$ for different CNTs.

\begin{tabular}{lcccc}
\hline CNT & $d(\AA)$ & $\lambda(\AA)$ & $r_{\text {eff }}(\AA)$ & $A_{\text {eff }}(\AA)$ \\
\hline$(5,5)$ & 6.8 & 2.8 & 0.6 & 1.1 \\
$(6,6)$ & 8.1 & 2.3 & 1.8 & 9.8 \\
$(7,7)$ & 9.5 & 2.3 & 2.5 & 18.8 \\
$(8,0)$ & 6.3 & 2.7 & 0.4 & 0.6 \\
$(10,0)$ & 7.9 & 2.9 & 1.0 & 3.2 \\
$(12,0)$ & 9.4 & 2.4 & 2.3 & 16.6 \\
\hline
\end{tabular}

of $(12,0)$ CNT is very low compared to the other CNTs with value of $0.1 \mathrm{eV}$. These values suggest that the interaction between water and armchair CNTs increases with diameter, but for zigzag CNTs it decreases with diameter.

Upon adding the water molecule to the CNTs, the band gap has been reduced dramatically in the armchair CNTs while remaining unchanged in the case of zigzag CNTs which reflects the fact that the interaction, taking place in the armchair CNTs, is much higher than that taking place in case of zigzag CNTs. The values of band gap for optimized geometry of CNT and water and the change of band gap and the interaction energy are shown in Table 3.

The interaction energy shows the expected trend obtained from hardness values. The calculated interaction energy increases with diameter for armchair CNTs, but it decreases 
TABLE 2: The energy of CNT, $E_{\mathrm{HOMO}}, E_{\mathrm{LUMO}}$, band gap, electronegativity, and hardness.

\begin{tabular}{|c|c|c|c|c|c|c|}
\hline CNT & $E(\mathrm{eV})$ & $E_{\text {HOMO }}(\mathrm{eV})$ & $E_{\text {LUMO }}(\mathrm{eV})$ & $\Delta E(\mathrm{eV})$ & $\chi(\mathrm{eV})$ & $\eta(\mathrm{eV})$ \\
\hline$(5,5)$ & -20625.723 & -7.037 & -1.885 & 5.152 & 4.461 & 2.576 \\
\hline$(6,6)$ & -24752.264 & -7.216 & -1.844 & 5.372 & 4.530 & 2.686 \\
\hline$(7,7)$ & -28878.628 & -7.329 & -1.829 & 5.500 & 4.579 & 2.750 \\
\hline$(8,0)$ & -33000.144 & -5.068 & -3.391 & 1.677 & 4.229 & 0.839 \\
\hline$(10,0)$ & -41253.453 & -5.139 & -3.664 & 1.475 & 4.401 & 0.737 \\
\hline$(12,0)$ & -49506.114 & -4.490 & -4.262 & 0.228 & 4.376 & 0.114 \\
\hline
\end{tabular}

TABLE 3: The energy of CNT with water, $E_{\mathrm{HOMO}}, E_{\mathrm{LUMO}}$, band gap, the interaction energy $\left(E_{\mathrm{int}}\right)$, and the change in band gap.

\begin{tabular}{|c|c|c|c|c|c|c|}
\hline $\mathrm{CNT}$ & $E_{\text {water+CNT }}(\mathrm{eV})$ & $\mathrm{HOMO}(\mathrm{eV})$ & LUMO $(\mathrm{eV})$ & $\Delta E(\mathrm{eV})$ & $E_{\text {int }}(\mathrm{Kcal} / \mathrm{mol})$ & $\delta \Delta E(\mathrm{eV})$ \\
\hline$(5,5)$ & -22693.537 & -4.467 & -1.923 & 2.544 & 3.634 & 2.608 \\
\hline$(6,6)$ & -26819.828 & -6.179 & -1.819 & 4.360 & -4.738 & 1.013 \\
\hline$(7,7)$ & -30946.226 & -6.604 & -1.818 & 4.786 & -5.543 & 0.714 \\
\hline$(8,0)$ & -35067.597 & -5.059 & -3.428 & 1.631 & -2.185 & 0.046 \\
\hline$(10,0)$ & -43320.898 & -5.138 & -3.667 & 1.471 & -2.001 & 0.004 \\
\hline$(12,0)$ & -51573.369 & -4.483 & -4.275 & 0.207 & 2.829 & 0.021 \\
\hline
\end{tabular}

TABLE 4: The required pressure to complete RO process.

\begin{tabular}{lccc}
\hline CNT & Diameter $(\AA)$ & $V\left(\AA^{3}\right)$ & $P(\mathrm{MPa})$ \\
\hline$(5,5)$ & 6.785 & 88.7503758 & 285 \\
$(6,6)$ & 8.142 & 127.8005411 & 258 \\
$(7,7)$ & 9.499 & 173.9507366 & 221 \\
$(8,0)$ & 6.268 & 139.7187241 & 109 \\
$(10,0)$ & 7.834 & 218.3105064 & 64 \\
$(12,0)$ & 9.401 & 314.3671292 & 62 \\
\hline
\end{tabular}

for zigzag CNTs. The interaction energy, hardness, and change in band gap show that the interaction of water with armchair CNTs is larger than that with zigzag CNTs. This suggests that zigzag CNTs are more efficient for RO water desalination process than armchair CNTs. The interaction energy between water and zigzag CNTs decreases with diameter from attractive in case of $(8,0) \mathrm{CNT}$ to repulsive in case of $(12,0)$ CNT.

The calculations for the required pressure to complete the RO process are summarized in Table 4 . The pressure was found to be in the range 200-300 MPa for the armchair CNTs, which is the value used in the molecular dynamics simulations to get accurate results without significant statistical errors [18]. The pressure for zigzag CNTs was found to be in the range of $60-110 \mathrm{MPa}$, which clearly verifies the same result, that zigzag CNTs are better for RO than armchair CNTs.

\section{Summary}

DFT calculation was performed to view the interaction between the CNT and water during the desalination process using CNT. It is shown that the water molecule suffers a strong repulsion at the wall neighborhood; few angstroms away from the wall, the water molecule has weak interaction that needs to be overcome by hydrostatic pressure of values in the range of $200 \mathrm{MPa}$ for armchair CNTs, and this value could be reduced to $60 \mathrm{MPa}$ when using zigzag CNTs. The calculation concludes that zigzag CNTs are more efficient for $\mathrm{RO}$ water desalination process over armchair CNTs.

Among zigzag CNTs, $(10,0)$ and $(12,0)$ CNTs might be more suitable for the desalination process than $(8,0) \mathrm{CNT}$ since they require less pressure and have a smaller change in band gap. The fact that as the diameter increases the salt rejection decreases provides that $(10,0)$ CNT is more selective for water than $(12,0)$ CNT. Molecular dynamics studies showed that $100 \%$ salt rejection for $(5,5)$ and $95 \%$ salt rejection for $(7,7)$ are realizable [18]. Also $(12,0)$ CNT has almost the same diameter as $(7,7)$ CNT suggesting a similar salt rejection behavior. The calculated pressure and change in band gap for $(12,0)$ and $(10,0)$ CNTs are very similar and much better than those of $(8,0) \mathrm{CNT}$, since the absolute value of the binding energy for $(10,0) \mathrm{CNT}$ is smaller than that of $(12,0)$ and $(8,0)$ CNTs. These data strongly recommend the use of $(10,0)$ CNT for water desalination process since it provides the weakest interaction with water among all the studied CNTs enabling a better flow rate of water inside the CNT.

\section{References}

[1] I. C. Karagiannis and P. G. Soldatos, "Water desalination cost literature: review and assessment," Desalination, vol. 223, no. 13, pp. 448-456, 2008.

[2] C. Fritzmann, J. Löwenberg, T. Wintgens, and T. Melin, "Stateof-the-art of reverse osmosis desalination," Desalination, vol. 216, no. 1-3, pp. 1-76, 2007.

[3] D. Wirth and C. Cabassud, "Water desalination using membrane distillation: comparison between inside/out and outside/in permeation," Desalination, vol. 147, no. 1-3, pp. 139-145, 2002.

[4] J. W. Mintmire and C. T. White, "Electronic and structural properties of carbon nanotubes," Carbon, vol. 33, no. 7, pp. 893902, 1995. 
[5] J.-P. Issi, L. Langer, J. Heremans, and C. H. Olk, "Electronic properties of carbon nanotubes: experimental results," Carbon, vol. 33, no. 7, pp. 941-948, 1995.

[6] C. Goze, L. Vaccarini, L. Henrard, P. Bernier, E. Hernandez, and A. Rubio, "Elastic and mechanical properties of carbon nanotubes," Synthetic Metals, vol. 103, no. 1-3, pp. 2500-2501, 1999.

[7] D. T. Mitchell, S. B. Lee, L. Trofin et al., "Smart nanotubes for bioseparations and biocatalysis," Journal of the American Chemical Society, vol. 124, no. 40, pp. 11864-11865, 2002.

[8] M. S. Dresselhaus, G. Dresselhaus, and R. Saito, "Physics of carbon nanotubes," Carbon, vol. 33, no. 7, pp. 883-891, 1995.

[9] S. Iijima, "Helical microtubules of graphitic carbon," Nature, vol. 354, no. 6348, pp. 56-58, 1991.

[10] A. A. Rafati, S. M. Hashemianzadeh, and Z. B. Nojini, "Effect of the adsorption of oxygen on electronic structures and geometrical parameters of armchair single-wall carbon nanotubes: a density functional study," Journal of Colloid and Interface Science, vol. 336, no. 1, pp. 1-12, 2009.

[11] P. Chen, X. Wu, J. Lin, and K. L. Tan, "High $\mathrm{H}_{2}$ uptake by alkalidoped carbon nanotubes under ambient pressure and moderate temperatures," Science, vol. 285, no. 5424, pp. 91-93, 1999.

[12] C. Liu, Y. Y. Fan, M. Liu, H. T. Cong, H. M. Cheng, and M. S. Dresselhaus, "Hydrogen storage in single-walled carbon nanotubes at room temperature," Science, vol. 286, no. 5442, pp. 1127-1129, 1999.

[13] A. C. Dillon, K. M. Jones, T. A. Bekkedahl, C. H. Kiang, D. S. Bethune, and M. J. Heben, "Storage of hydrogen in single-walled carbon nanotubes," Nature, vol. 386, no. 6623, pp. 377-379, 1997.

[14] J. Zhao, A. Buldum, J. Han, and J. P. Lu, "Gas molecule adsorption in carbon nanotubes and nanotube bundles," Nanotechnology, vol. 13, no. 2, pp. 195-200, 2002.

[15] C. Tang, Q. Zhang, K. Wang, Q. Fu, and C. Zhang, "Water transport behavior of chitosan porous membranes containing multi-walled carbon nanotubes (MWNTs)," Journal of Membrane Science, vol. 337, no. 1-2, pp. 240-247, 2009.

[16] G. Hummer, J. C. Rasaiah, and J. P. Noworyta, "Water conduction through the hydrophobic channel of a carbon nanotube," Nature, vol. 414, no. 6860, pp. 188-190, 2001.

[17] J. A. Thomas, A. J. H. McGaughey, and O. Kuter-Arnebeck, "Pressure-driven water flow through carbon nanotubes: Insights from molecular dynamics simulation," International Journal of Thermal Sciences, vol. 49, no. 2, pp. 281-289, 2010.

[18] B. Corry, "Designing carbon nanotube membranes for efficient water desalination," Journal of Physical Chemistry B, vol. 112, no. 5, pp. 1427-1434, 2008.

[19] W. Humphrey, A. Dalke, and K. Schulten, "VMD: visual molecular dynamics," Journal of Molecular Graphics, vol. 14, no. 1, pp. 33-38, 1996.

[20] A. D. Becke, "Density-functional exchange-energy approximation with correct asymptotic behavior," Physical Review A, vol. 38, no. 6, pp. 3098-3100, 1988.

[21] A. D. Becke, "Density-functional thermochemistry. III. The role of exact exchange," The Journal of Chemical Physics, vol. 98, no. 7, pp. 5648-5652, 1993.

[22] C. Lee, W. Yang, and R. G. Parr, "Development of the ColleSalvetti correlation-energy formula into a functional of the electron density," Physical Review B, vol. 37, no. 2, pp. 785-789, 1988.

[23] B. Miehlich, A. Savin, H. Stoll, and H. Preuss, "Results obtained with the correlation energy density functionals of becke and
Lee, Yang and Parr," Chemical Physics Letters, vol. 157, no. 3, pp. 200-206, 1989.

[24] J. M. L. Martin, J. El-Yazal, and J. François, "Basis set convergence and performance of density functional theory including exact exchange contributions for geometries and harmonic frequencies," Molecular Physics, vol. 86, pp. 1437-1450, 1995.

[25] M. J. Frisch, G. W. Trucks, H. B. Schlegel et al., Gaussian 09, Revision A. 02, Gaussian, Wallingford, Conn, USA, 2009.

[26] R. Dennington, T. Keith, and J. Millam, GaussView, Version 5, Semichem, Shawnee Mission, Kan, USA, 2009.

[27] J. Charlier, X. Blase, and S. Roche, "Electronic and transport properties of nanotubes," Reviews of Modern Physics, vol. 79, no. 2, pp. 677-732, 2007.

[28] R. G. Parr and W. Yang, "Density functional approach to the frontier-electron theory of chemical reactivity," Journal of the American Chemical Society, vol. 106, no. 14, pp. 4049-4050, 1984.

[29] R. G. Pearson, "Hard and soft acids and bases," Journal of the American Chemical Society, vol. 85, no. 22, pp. 3533-3539, 1963.

[30] R. G. Pearson, “The principle of maximum hardness," Accounts of Chemical Research, vol. 26, no. 5, pp. 250-255, 1993. 

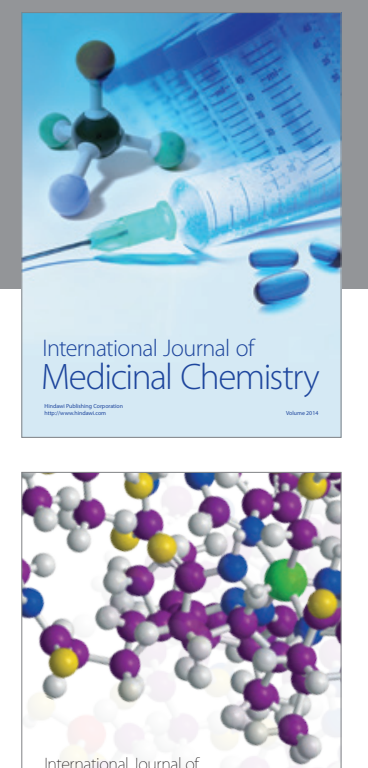

\section{Carbohydrate} Chemistry

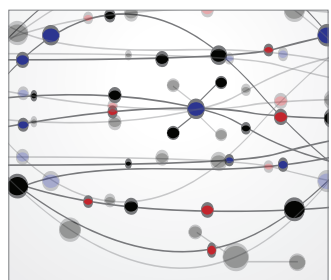

The Scientific World Journal
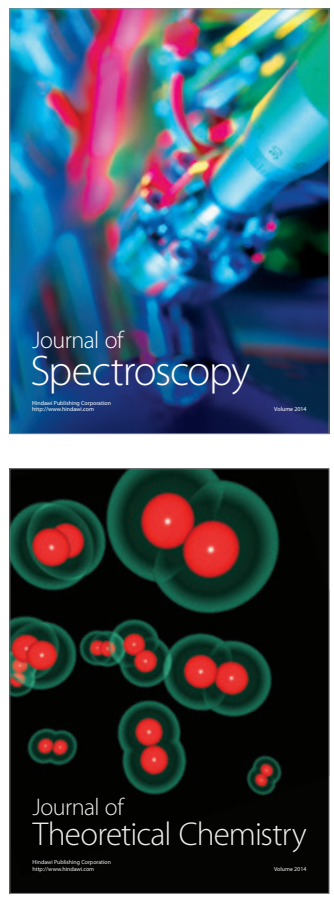
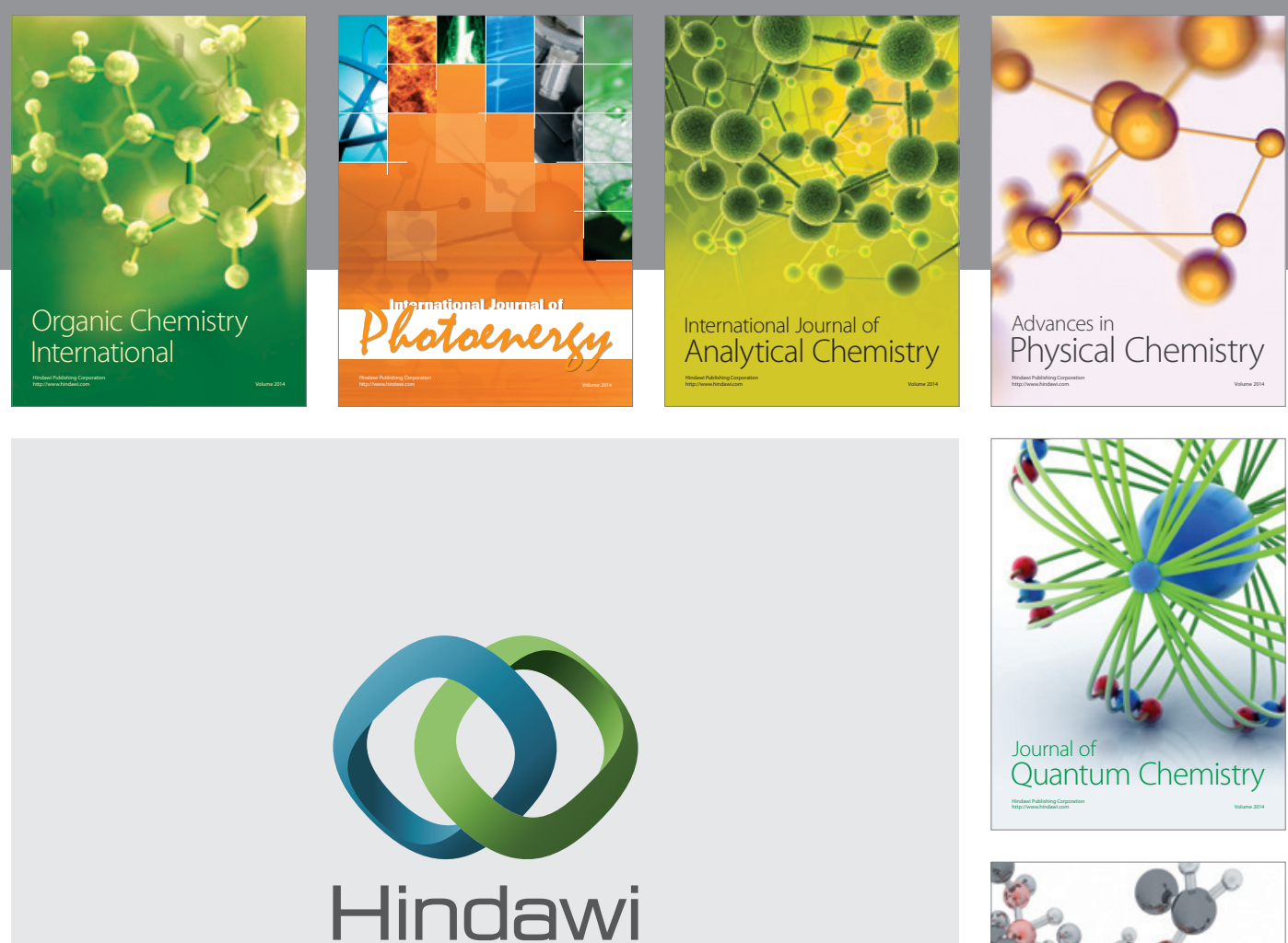

Submit your manuscripts at

http://www.hindawi.com

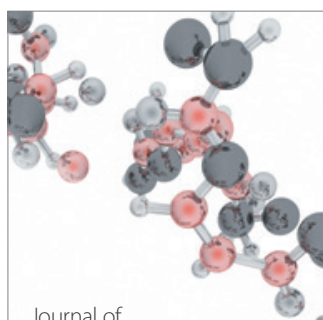

Analytical Methods

in Chemistry

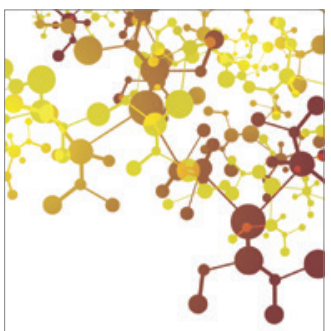

Journal of

Applied Chemistry

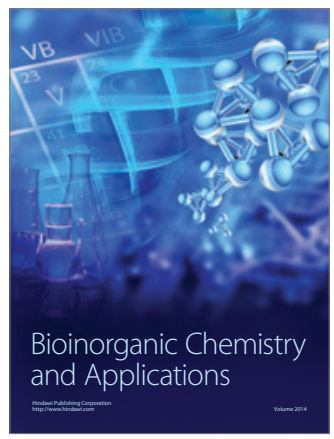

Inorganic Chemistry
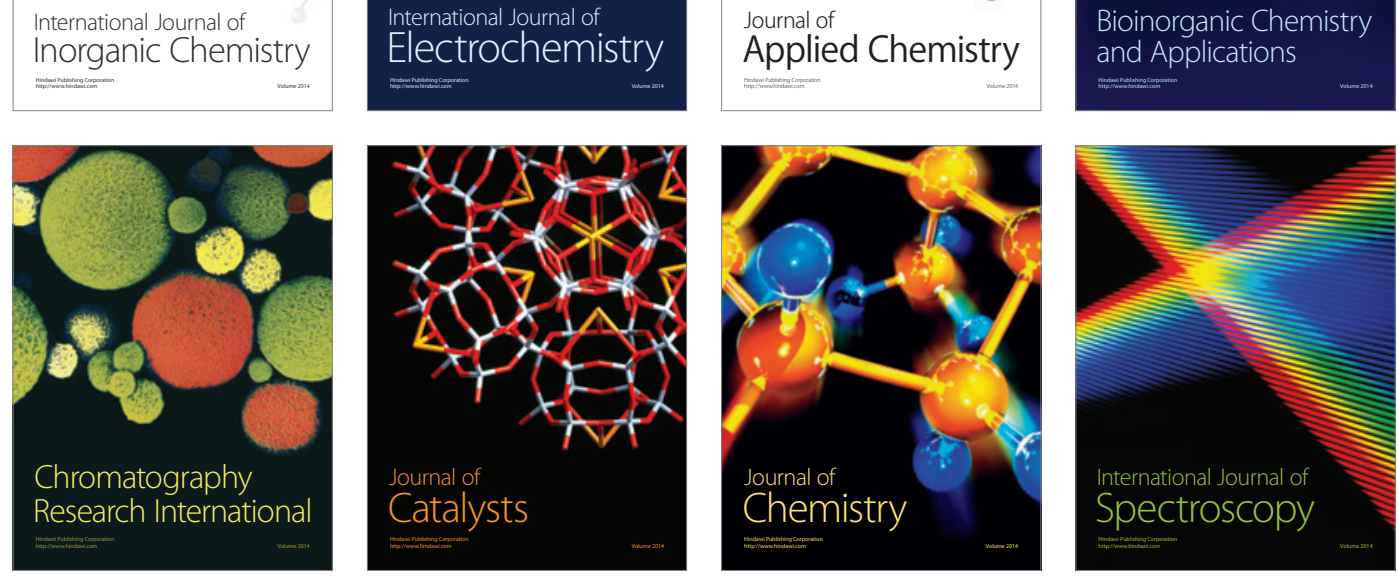